\title{
Four Algorithms for Boundary Control with Breaking in Space and Time
}

\author{
Vladimir Arabadzhi \\ Division of Geophysical Research, Institute of Applied Physics, Nizhny Novgorod, Russian \\ Email: v.v.arabadzhi@appl.sci-nnov.ru
}

How to cite this paper: Arabadzhi, V. (2019) Four Algorithms for Boundary Control with Breaking in Space and Time. Journal of Applied Mathematics and Physics, 7, 2891-2901.

https://doi.org/10.4236/jamp.2019.711198

Received: October 18, 2019

Accepted: November 24, 2019

Published: November 27, 2019

\begin{abstract}
Typically, active control systems either have a priori complete information about the boundary-value problem and damped waves before switching on, or get it during the measurement process or accumulate and update information online (identification process in adaptive systems). In this case, the boundary problem is completely imprinted in the information arrays of the control system. However, very often complete information about a boundary-value problem is not available in principle or this info is changing in time faster than the process of its accumulation. The article considers examples of boundary control algorithms based almost without any information. The algorithms presented in the article cannot be obtained within the framework of the harmonic representation of the problem by complex amplitudes. And these algorithms carry out fast control in microstructured boundary problems. It is shown that in some cases it is possible to find simple solutions if we remove restrictions: 1) on the spatio-temporal resolution of controlling elements of a boundary-value problem; 2) on the high-frequency radiation of the controlled boundary.
\end{abstract}

\section{Keywords}

Incident Low Frequency Wave, High Frequency Technological Radiation, Fast Control in Microstructured Boundary Problems, Binary Breaker, Breaker-Inverter, Length of Damping, Spinning Acoustic Blades, Gas Stream, Seiche Waves

\section{Introduction}

With constant (in time) parameters (or without frequency conversion) devices for wideband non-resonant sound suppression (or other types of waves) should have large wave sizes (thickness $\ell_{D}$, see Figure 1(a)) of the order of the maxi- 
mum wavelength $\lambda_{\mathrm{w}}$ of the frequency range of suppression $\lambda_{\min }<\lambda_{\mathrm{w}}<\lambda_{\max }$ (where $\left.\lambda_{\min } / \lambda_{\max }<<1\right)$ i.e. $\ell_{\mathrm{D}} \geq \lambda_{\max }$. The goal of this work is to reduce the dimensions of broadband (non-resonant) suppression devices (for cancellation, damping, absorption, suppression of reflections, ...) at minimum information on the boundary problem and on the wave to be damped. If we allow the arbitrary power of high-frequency sound radiation (technological radiation on the technological frequencies $\mathrm{f} \geq 1 / T$ ) and a rapid (on time scale $T$ ) change of parameters of a boundary value problem, then in several cases the goals set above can be achieved jointly. Below we will consider some simple applications of this approach. The examples of boundary control considered in the one-dimensional wave problem considered below are reduced to the alternation (in time) of two different types of the boundary-value problem. Jumping (switching) from one boundary-value problem to another and the connection between them provides a certain "breaker" controlled by the algorithm.

We mean breaking as a very quick and microscopic hop (jump) from one boundary value problem to another. Below we want to obtain: (a) effective suppression of long wave reflections (Figure 1(a), Figure 1(b)) from a controlled boundary $x=x_{\mathrm{b}}$ (and suppression of sound propagation in a gas stream too (Section 5), see Figure 1(c)); (b) in a wide band $\lambda_{\min }<<\lambda_{\max }$ of wavelengths; (c) without accumulation of information about the damped wave and the boundary value problem; (d) at small wave sizes of the suppressing device, i.e. $\ell_{\mathrm{D}}<<\lambda_{\min }$, due to relaxation (dissipation) of the technological waves on high frequencies $\mathrm{f} \geq 1 / T$ (i.e. $\left.\ell_{\mathrm{D}}=\ell_{\mathrm{D}}(\mathrm{f})=\ell_{\mathrm{D}}(1 / T)\right)$ is length of damping.

\section{Algorithm of Half-Return of the Boundary (AHRB)}

The goal of the algorithm is to suppress reflections from boundary $x_{\mathrm{b}}$. We consider a semi-infinite $\left(x_{\mathrm{b}} \leq x<\infty\right)$ elastic rod, with longitudinal impedance $Z$,

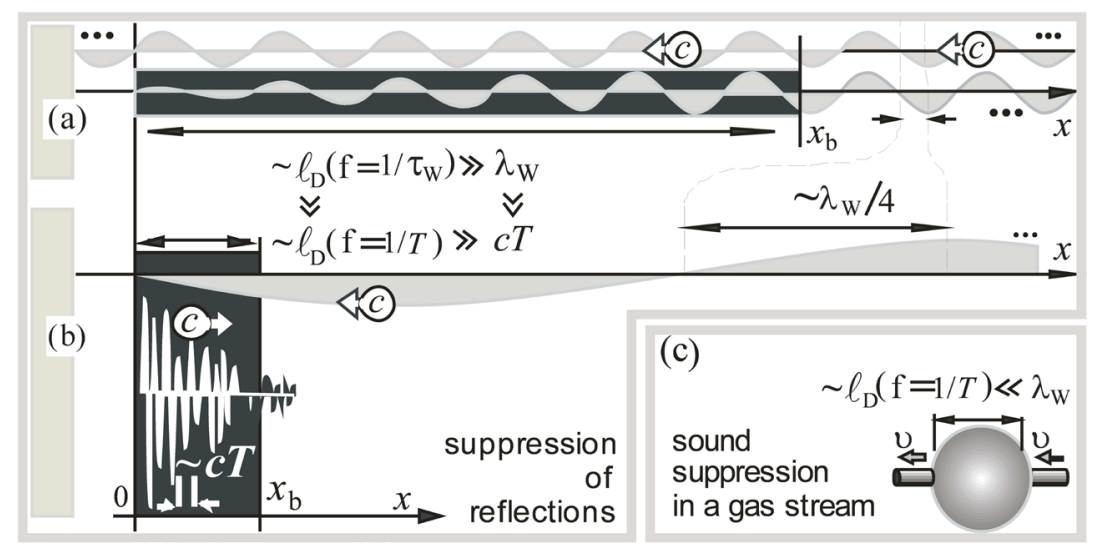

Figure 1. General statement of the problem and goals of approach: black area means suppressing layer of thickness $\ell_{\mathrm{D}}$ (traditional thick wideband suppressing layer with parameters constant in time (a) and thin wideband suppressing layer with high frequency operations (b)); white-black wave (b) means reflected high frequency waves conversed from incident one (gray wave means incident wave); (c) small size muffler for gas stream with long sound waves. 
sound speed $c$ of waves and the field $U(x, t)$ of longitudinal displacement of particles (Figure 2(a)). The boundary condition at the end $x_{\mathrm{b}}$ has the form $(\varepsilon S)[\partial U(x, t) / \partial x]_{x=x_{\mathrm{b}}}=F_{\mathrm{b}}(t)$, where $F_{\mathrm{b}}(t)$ is the force applied to end $x_{\mathrm{b}}, S$ is the cross-section of the rod, $\varepsilon$ is Young's modulus. A smooth incident wave $U(x, t)=U_{\mathrm{W}}(x+c t) \quad$ (temporal scale $\left.\tau_{\mathrm{W}}\right)$ runs from the right. On the free end $x_{\mathrm{b}} \quad\left(\right.$ at $\left.\quad F_{\mathrm{b}}=0\right)$ we have $U_{\mathrm{b}}(t)=2 U_{\mathrm{W}}\left(x_{\mathrm{b}}+c t\right)$ and $U_{\mathrm{b}}(t)-U_{\mathrm{b}}(t-\tau) \approx 2 \tau\left[U_{\mathrm{W}}\left(x_{\mathrm{b}}+c t\right)\right]_{t}^{\prime}$ for any interval $\tau<<\tau_{W}$.

The boundary-value problem can be represented as the sum of two partial linear problems: (a1) reflection of the incident wave (IW) $U_{\mathrm{W}} \neq 0$ from the free (at $F_{\mathrm{b}}=0$ ) the end of the rod; (a2) wave generation by force $F_{\mathrm{b}} \neq 0$ in the absence of an incident wave (at $\left.U_{\mathrm{W}}=0\right)$. The "breaker" jumps $((\mathrm{a} 1) \leftrightarrow(\mathrm{a} 2))$ in accordance with the control algorithm: measurement in (a1), action in (a2). We assume that the force $F_{\mathrm{b}}$ has a compact support: $\left|F_{\mathrm{b}}\right|>0$ at $t \in\left[0, \tau_{\mathrm{F}}\right]$, $F_{\mathrm{b}}=0$ at $t \notin\left[0, \tau_{\mathrm{F}}\right]$. For a clear distinction between the causes and consequences in the work of AHRB, it is extremely important that after the termination of the force $F_{\mathrm{b}}$ (at $t>\tau_{\mathrm{F}}$ ), the displacement $\int_{0}^{\tau_{\mathrm{F}}} Z^{-1} F_{\mathrm{b}}(\xi) d \xi$ of the boundary $x_{\mathrm{b}}$ caused by this force is saved indefinitely long [1] after its switching off. Now we will directly consider AHRB, which is a sequence of time cycles (see Figure 2(c)) of the boundary control. Each $n$-th cycle $t_{\mathrm{n}-1} \leq t<t_{\mathrm{n}-1}+T_{\mathrm{n}}$ of duration $T_{\mathrm{n}}(\mathrm{n}=0,1,2, \ldots)$ consists of two parts: (a) smooth displacement of the free (i.e. at $F_{\mathrm{b}}=0$ ) end $x_{\mathrm{b}}$ over a time interval $t_{\mathrm{n}-1} \leq t<t_{\mathrm{n}-1}+\left(\tau_{\mathrm{f}}\right)_{\mathrm{n}}$, we set the duration $\left(\tau_{\mathrm{f}}\right)_{\mathrm{n}}$ of this interval arbitrary under condition $\left(\tau_{\mathrm{f}}\right)_{\mathrm{n}}<<\tau_{\mathrm{W}}$, and we measure the corresponding displacement $\left[U_{\mathrm{b}}\left(t_{\mathrm{n}-1}+\left(\tau_{\mathrm{f}}\right)_{\mathrm{n}}\right)-U_{\mathrm{b}}\left(t_{\mathrm{n}-1}\right)\right]$ of boundary $x_{\mathrm{b}}$; (b) the rapid return of the border $x_{\mathrm{b}}$ during the interval $t_{\mathrm{n}-1}+\left(\tau_{\mathrm{f}}\right)_{\mathrm{n}} \leq t<t_{\mathrm{n}-1}+\left(\tau_{\mathrm{f}}\right)_{\mathrm{n}}+\left(\tau_{\mathrm{r}}\right)_{\mathrm{n}}$ under the action of force $F_{\mathrm{b}}$. Force $F_{\mathrm{b}}(t)$ is switched off at the moment $t=t_{\mathrm{n}-1}+\left(\tau_{\mathrm{f}}\right)_{\mathrm{n}}+\left(\tau_{\mathrm{r}}\right)_{\mathrm{n}}$ when the level $-\left[U_{\mathrm{b}}\left(t_{\mathrm{n}-1}+\left(\tau_{\mathrm{f}}\right)_{\mathrm{n}}\right)-U_{\mathrm{b}}\left(t_{\mathrm{n}-1}\right)\right] / 2$ becomes crossed by function

$\varphi[\xi]=\int_{t_{\mathrm{n}-1}+\left(\tau_{\mathrm{f}}\right)_{\mathrm{n}}}^{t_{\mathrm{n}-1}+\left(\tau_{\mathrm{f}}\right)_{\mathrm{n}}+\xi} Z^{-1} F_{\mathrm{b}}(\eta) d \eta$ for the first time (at the value $\left.\xi=\left(\tau_{\mathrm{r}}\right)_{\mathrm{n}}\right)$ beginning from the moment $t=t_{\mathrm{n}-1}+\left(\tau_{\mathrm{f}}\right)_{\mathrm{n}}$. It is easy to see from the Figure 2(c) than when the scale $T$ of control cycle, scale $\tau_{\mathrm{f}}\left(\left(\tau_{\mathrm{f}}\right)_{\mathrm{n}} \sim \tau_{\mathrm{f}}\right)$ of free drift, scale $\tau_{\mathrm{r}}$ $\left(\left(\tau_{\mathrm{r}}\right)_{\mathrm{n}} \sim \tau_{\mathrm{r}}\right)$ of half-return, scale $\tau_{\mathrm{W}}$ of IW are satisfying the condition $\tau_{\mathrm{r}}<<\tau_{\mathrm{f}}<<\tau_{\mathrm{W}}$, we get the weakness of reflections on the frequencies $\mathrm{f} \sim 1 / \tau_{\mathrm{W}}$ or $U_{\mathrm{b}}(t) \rightarrow U_{\mathrm{W}}\left(x_{\mathrm{b}}+c t\right)$ and $\left|U_{\mathrm{b}}(t)-U_{\mathrm{W}}\left(x_{\mathrm{b}}+c t\right)\right| / \bar{U}_{\mathrm{W}} \rightarrow 0$, where

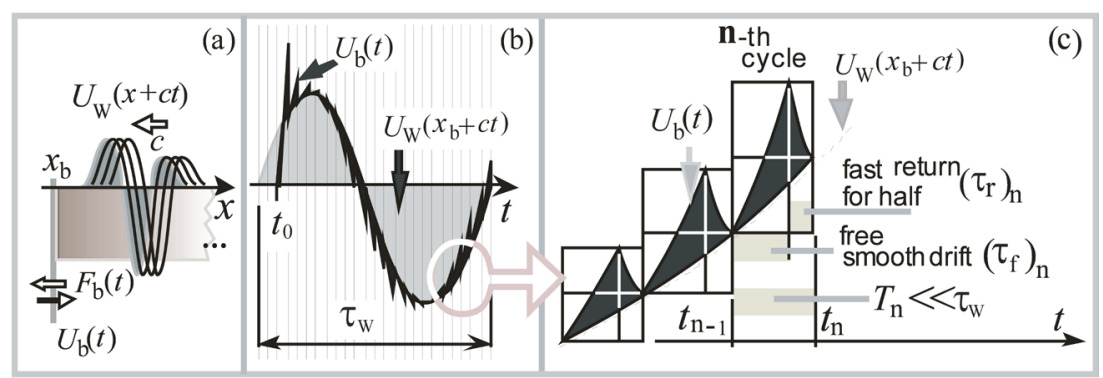

Figure 2. To AHRB: (a) geometry of control problem; functional intervals of AHRB (b), (c). 
$\bar{U}_{\mathrm{W}}=\left(1 / \tau_{\mathrm{W}} c\right) \int_{0}^{\tau_{\mathrm{W}} c}\left|U_{\mathrm{W}}(\xi)\right| d \xi$. The peak $\hat{P}_{\mathrm{hf}}$ and average $\bar{P}_{\mathrm{hf}} \quad$ (on the cycle $\sim T$ ) power of the high-frequency radiation, generated by the impact force $F_{\mathrm{b}}$, and time averaged power flux $\bar{P}_{\mathrm{W}}$ in the low frequency IW are satisfying to the following relations $\hat{P}_{\mathrm{hf}} / \bar{P}_{\mathrm{W}} \approx\left(\tau_{\mathrm{f}} / \tau_{\mathrm{r}}\right)^{2}>>1$ and $\bar{P}_{\mathrm{hf}} / \bar{P}_{\mathrm{W}} \approx \tau_{\mathrm{f}} / \tau_{\mathrm{r}}>>1$. About impedance: $Z$ is unknown and can slowly change in time $Z(t)$. About impact force: $F_{\mathrm{b}}(t)$ can be of arbitrary pulse shape, but with constant sign and at any moment of impact satisfies the condition $\bar{F}_{\mathrm{b}} \gg \bar{\Psi}_{\mathrm{w}}$, where

$\bar{F}_{\mathrm{b}}=\left(1 / \tau_{\mathrm{w}}\right) \int_{0}^{\tau_{\mathrm{W}}} F_{\mathrm{b}}(t) d t, \quad \bar{\Psi}_{\mathrm{w}}=\left(\varepsilon S / \tau_{\mathrm{W}}\right)^{-1} \int_{0}^{\tau_{\mathrm{W}} c}\left|\left(U_{\mathrm{W}}(x+c t)\right)_{x}^{\prime}\right| d x$. About linearity: the returning path scale is $h=\left(\tau_{\mathrm{f}} / \tau_{\mathrm{W}}\right) \bar{U}_{\mathrm{W}}$, velocity scale is $h / \tau_{\mathrm{r}}$, so the condition $h / \tau_{\mathrm{r}}<<c$ ensures linearity.

\section{Algorithm of Maximum Instant Power Absorbed (AMIP)}

The goal of the algorithm AMIP is to maximize the instantaneous power absorbed by the boundary $x_{\mathrm{b}}$. Consider above rod problem: some electric drive (as above "breaker") can ensure any constant velocity $V_{\mathrm{b}}$ of edge $x_{\mathrm{b}}$ independently of any incident wave (IW). Wave problem can be represented as the sum of two linear problems: (a1) reflection of IW from a fixed $\left(V_{\mathrm{b}}=d U_{\mathrm{b}} / d t=0\right)$ boundary $x_{\mathrm{b}}$; (a2) the radiation of waves by a boundary $x_{\mathrm{b}}$ at a given velocity $V_{\mathrm{b}}$ in the absence of IW. The breaker jumps: (a1) $\leftrightarrow$ (a2). Velocity $V_{\mathrm{b}}$ takes discrete levels $V_{\mathrm{b}}\left(t_{\mathrm{n}}\right)=V_{\mathrm{n}}$ at discrete time intervals $(\mathrm{n}-1) T<t<\mathrm{n} T \quad(\mathrm{n}=1,2, \ldots)$, where $T$ is the period of velocity switching (and measuring between switching). Steps $V_{\mathrm{n}}$ are multiples of the tuning step $\bar{V}, V_{\mathrm{n}} / \bar{V}=0, \pm 1, \pm 2, \pm 3, \ldots$ (integer).

Absorbed power (work of IW with a border $x_{\mathrm{b}}$ ) is square function $W(t)=V_{\mathrm{b}}(t)\left[2 E_{\mathrm{W}}\left(x_{\mathrm{b}}+c t\right)-Z V_{\mathrm{b}}(t)\right]$ of $V(t)$ with the unique maximum at $V_{\mathrm{b}}(t)=V_{\mathrm{W}}\left(x_{\mathrm{b}}+c t\right)=E_{\mathrm{W}}\left(x_{\mathrm{b}}+c t\right) / Z, \quad$ where $Z=\operatorname{Re} Z, \quad V_{\mathrm{w}}\left(x_{\mathrm{b}}+c t\right) \quad$ and $E_{\mathrm{W}}\left(x_{\mathrm{b}}+c t\right)$ are longitudinal particle velocity and stress in IW in infinite rod. AMIP is expressed by the iterative (recurrent) relation $V_{\mathrm{n}}=V_{\mathrm{n}-1}+\bar{V} \operatorname{sgn}(\bar{W})$ (for $\mathrm{n} \geq 2$ with initial condition $V_{1}=0, V_{1}=\bar{V}$ ), where: $\bar{W}=W_{\mathrm{n}-1}-W_{\mathrm{n}-2}$, $W_{\mathrm{n}-1}=F_{\mathrm{n}-1} V_{\mathrm{n}-1}, W_{\mathrm{n}-2}=F_{\mathrm{n}-2} V_{\mathrm{n}-2} ; F_{\mathrm{n}-1}, F_{\mathrm{n}-2}$ are measured values of the force applied to boundary $x_{\mathrm{b}}$ by the medium of rod from $x \geq x_{\mathrm{b}}$ at the moments $t_{\mathrm{n}-1}-a, \quad t_{\mathrm{n}-2}-a$ correspondingly $(0<a<<T) ; \operatorname{sgn}(\xi)=+1$ at $\xi>0$, $\operatorname{sgn}(\xi)=-1$ at $\xi<0$. If at the previous step the velocity increase causes the decrease $(\bar{W}<0)$ of the absorbed power, at the next step the velocity increase will change its sign and will not change it in the opposite case. In above one dimensional statement of the problem absorption maximum corresponds to the minimum of reflection and radiation too. AMIP does not need to know either rod impedance $Z$ and IW. AMIP effectively traces IW if the following conditions are satisfied: $\bar{V}<<\max \left|\partial U_{\mathrm{w}} / \partial t\right|, \bar{V} / T>>\max \left|\partial^{2} U_{\mathrm{W}} /(\partial t)^{2}\right|$ or $\tau_{\mathrm{W}} \bar{V}<<\bar{U}_{\mathrm{W}}<<\left(\tau_{\mathrm{W}}\right)^{2} T^{-1} \bar{V}$, AMIP resembles the algorithm of random search, considered in [2]. Weak boundary radiation on frequencies $\mathrm{f}=\mathrm{n} / T \quad(\mathrm{n}=1,2,3, \ldots)$ is defined by the scale of the velocity-tuning step $T$. The average power $\left\langle P_{\mathrm{hf}}\right\rangle_{T}$ over the interval $T$ of high-frequency (at frequencies $1 / T, 2 / T, 2 / T, \ldots$ ) radiation of the boundary $x=x_{\mathrm{b}}$ is of the order $\left\langle P_{\mathrm{hf}}>_{\mathrm{T}} \approx(\bar{V})^{2} Z\right.$. AMIP can 
also be applied in the problem of IW absorbing in a thin infinite elastic plate (see Figure 3(b)), since in this case the impedance of the plate with respect to a point source of normal velocity is also purely $\operatorname{real}(Z=\operatorname{Re} Z$, [1]). Thus, by adjusting the normal velocity $V_{\mathrm{b}}(t)$ of the point $\mathbf{r}_{\mathrm{b}}$ of application of external force to the maximum instantaneous absorbed power, it is possible to achieve the maximum absorption cross section $\sigma=\lambda_{\mathrm{W}} / 2 \pi$ for a point source of the normal plate velocity at the point $\mathbf{r}_{b}$.

\section{Algorithm for Boundary Condition Modulation (ABCM)}

The goal of the algorithm is to suppress reflections from boundary $x_{\mathrm{b}}$ in the above one-dimensional problem and in layer (or distance from $x_{\mathrm{b}}$ ) of small thickness at minimum info on IW and boundary problem. The ABCM is based on two main states of a controlled boundary $x_{\mathrm{b}}$ (Figure 4(a)): (a1) rigid state $\left[U_{t}^{\prime}(x, t)\right]_{x=x_{\mathrm{b}}}=0$ with a fixed boundary $x_{\mathrm{b}}$ and velocity reflection coefficient $\mathbf{R}=-1$; (a2) soft state $\left[U_{x}^{\prime}(x, t)\right]_{x=x_{\mathrm{b}}}=0$ with a free boundary $x_{\mathrm{b}}$ and velocity reflection coefficient $\mathbf{R}=+1$. Binary breaker $\mathbf{B}(t)$ jumps ((a1) $\leftrightarrow$ (a2)) with period $T<<\tau_{\mathrm{W}}$ and without doing work (without radiation or absorption) and without any measurements. ABCM algorithm controls the boundary condition $\alpha(t)\left[U_{x}^{\prime}(x, t)\right]_{x=x_{\mathrm{b}}}+\beta(t)\left[U_{t}^{\prime}(x, t)\right]_{x=x_{\mathrm{b}}}=0$ via the coefficients $\alpha, \beta$ (Figure 4(b)): [ $\mathbf{B}=1, \alpha=0, \beta=1, \mathbf{R}=-1] \leftrightarrow[\mathbf{B}=0, \alpha=1, \beta=0, \mathbf{R}=+1]$. As a result of such control (see Figure 4(c)), we obtain an oscillogram $V_{\mathrm{b}}(t)$ of the velocity of the boundary $V_{\mathrm{b}}(t)$, which on average (over a period $T$ ) tends to the velocity of particles in the incident wave (i.e., in an infinite rod without reflections) or $V_{\mathrm{b}}(t) \rightarrow V_{\mathrm{W}}\left(x_{\mathrm{b}}+c t\right)$ [3], as was required above. An experimental verification of the $\mathrm{ABCM}$ algorithm is presented below in Section 6. In this case, the boundary $x_{\mathrm{b}}$ converts the low-frequency (with a spatial scale $c \tau_{\mathrm{W}}$ ) IW
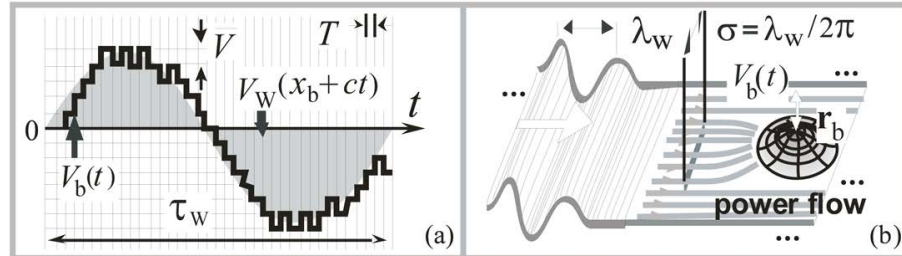

Figure 3. To the algorithm AMIP: boundary velocity and particle velocity in incident wave (a); on the absorption of bending wave in thin infinite elastic plate (b).

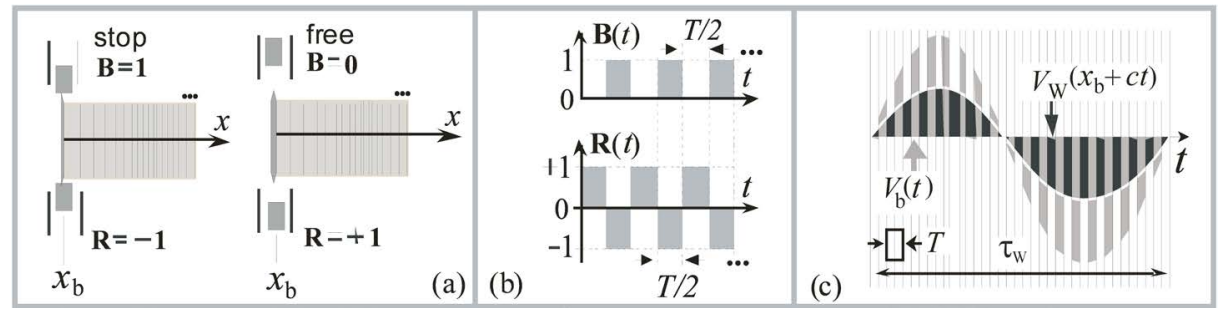

Figure 4. To the algorithm ABCM: breaker and boundary problem statement (a). States of breaker and reflection coefficient (b) boundary condition control (b). Velocity of controlled boundary and particle velocity in the incident wave (c). 
wave into reflected high-frequency waves at frequencies $\mathrm{f}=1 / T, 2 / T, 3 / T, \ldots$ that dissipatively attenuate in $\exp \left[-\ell / \ell_{\mathrm{D}}(\mathrm{f})\right]$ times at a distance $\ell$ from the boundary $x_{\mathrm{b}}$, where $\ell_{\mathrm{D}}=\ell_{\mathrm{D}}(\mathrm{f})$ is the frequency dependent dissipative attenuation length or equivalent damping device size (see Figure 1(b)).

Thus, having fulfilled the condition $c T<<\ell_{\mathrm{D}}(1 / T)<<\tau_{\mathrm{W}} c<<\ell_{\mathrm{D}}\left(1 / \tau_{\mathrm{W}}\right)$, it is possible to ensure the smallness of the attenuation length and smallness of effect of dissipation on the above boundary condition.

\section{Algorithm for Sound Blocking in the Gas Stream (ASBG)}

The goal of ASBG is to block the sound propagation in a gas stream with average velocity $v<<c$. This problem arises in the design of automobile silencers (Figure $1(\mathrm{c})$ ): we need a device that transmits a gas stream, but does not allow sound propagation in the gas. Traditionally (with parameters constant in time), there are two directions in this problem. On the one hand, pushing of gas through a grid of holes in a rigid plane. The smaller the diameters of the holes, the lower the sound transmission. This approach allows a small size $\ell_{\mathrm{D}}<<\lambda_{\min }$ of the silencer. But energy losses due to pushing gas through holes increase too much. On the other hand, passing a gas stream with sound through a low pass filter (Helholtz resonator) with resonance at wavelength $\lambda>\lambda_{\max }$ (with dimensions $\left.\ell_{\mathrm{D}}>\lambda_{\max }\right)$. This approach doesn't require power losses for gas pushing, but requires too large dimensions of muffler. Known silencers are usually a combination of approaches (a), (b) or a complicated combination of tubes, perforated plates and resonators. An approach below (based on a quick switching of parameters) allows dimensions $\ell_{\mathrm{D}}<<\lambda_{\min }$ with small power losses for pushing gas through silencer.

Consider the 1D case (Figure 5(a1)) of a gas stream (together with sound waves in it) from left to right between two rigid planes (waveguide). The binary breaker $\mathbf{B}(t)$ jumps (without doing any work with flow or wave field) from one boundary problem (state $\mathbf{B}=0$ ) to another (state $\mathbf{B}=1$ ) in accordance with the control algorithm periodically in time (without any measurements). Here $2 T$ is the period of states repetition, and $T$ is the period of states change, respectively. In even time intervals (of duration $T$ ) between two sections at a distance $L$ from each other, rigid thin flat walls arise instantaneously and simultaneously (state $\mathbf{B}=1$ ) and block the flow and sound. In odd intervals (over time $T=L / c$ ) of time, these walls also instaneously and simultaneously disappear (state $\mathbf{B}=0$ ). At the same time, a segment of a sound of length $L$ that is in a closed segment of length $L$ manages fully reverses (this is the goal of inverter-breaker $\mathbf{B}(t)=[0,1]$ works) just before the opening of the space interval. Thus, the inverter-breaker $\mathbf{B}(t)$ during half-time reflects (blocks sound) the sound back, and during another half time the stream passes or doesn't pass through itself disturbances propagating with the speed $c$ of sound. The disadvantages of this option are: (a) large power losses due to the production of sound from hydraulic impact; (b) the possibility of not a 1D sound propagation in waveguide. 


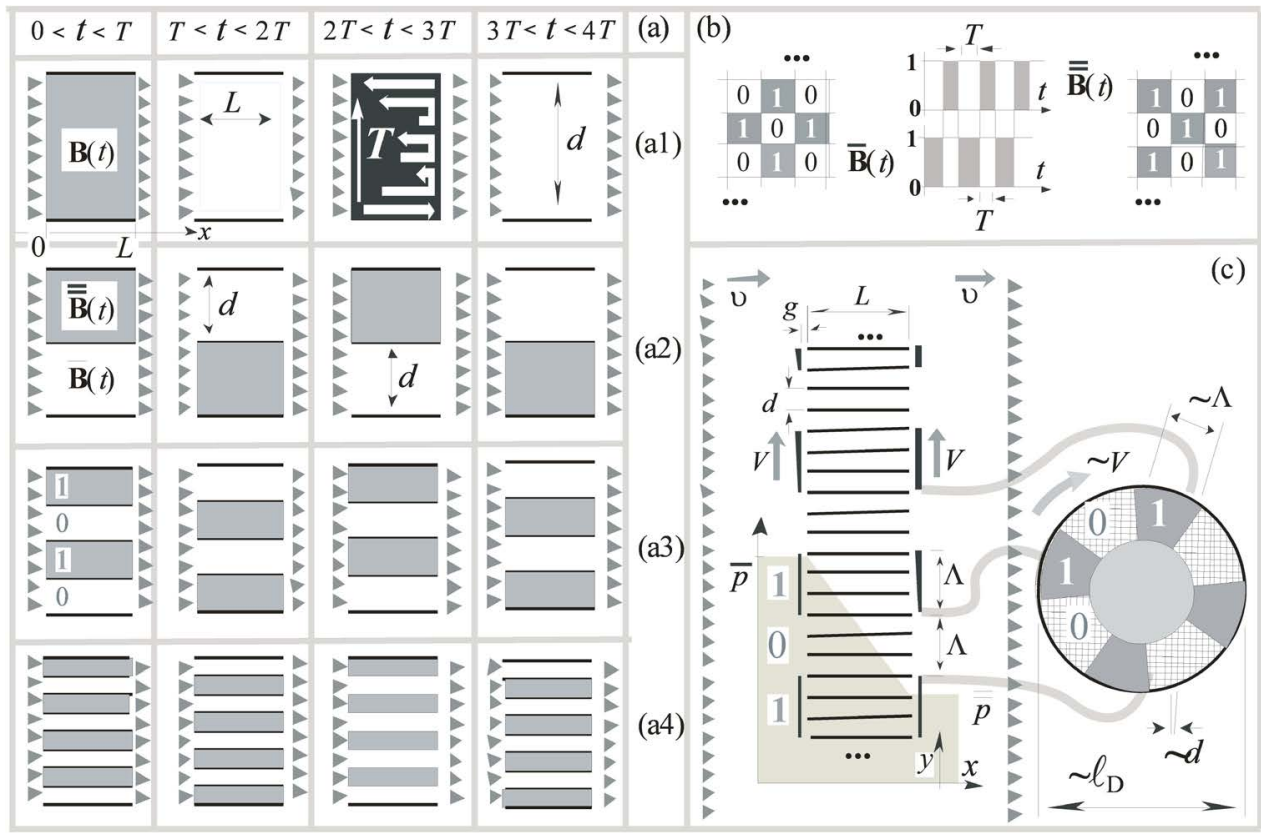

Figure 5. On the action of ASBG: (a) temporal sequence of states ("1"-closed state and "0"-opened state) of breakers-inverters at their growing amount ((a1), for 1 waveguide), (a2) 2 waveguides, (a3) 4 waveguides), (a4) 8 waveguides); (b) complementary breakers for the cross section of 3-D problem; (c) running acoustic blades (petals) and spinner.

Next are a lot (Figures 5(a2)-(a4)) of inverters-breakers in the system cross section, now a pair of mutually complementary $(\overline{\mathbf{B}}(t) \overline{\overline{\mathbf{B}}}(t)=0)$ inverter breakers $\overline{\mathbf{B}}(t), \overline{\overline{\mathbf{B}}}(t) \quad$ (see Figure 5(b)) with the corresponding time diagrams. The more elements in the cross section (Figures 5(a2)-(a4)), the weaker the hydraulic shock (flow energy $\rightarrow$ sound) when switching inverters and faster this blow becomes blurred (spatially averaged), helping to push the gas into neighboring opened waveguides. The above (evolution in Figure 5(a)) model is difficult to implement (it is not clear how it would be possible to create rapidly appearing and disappearing walls of inverter-breaker). However, the model shown in Figure 5(c) is much simpler to implement. Consider a 2-D echelon of thin infinite parallel rigid plane strips with a length $L$ and with a distance $d<<L$ between them. To the left, a gas flow at time averaged velocity enters this system (and exits with the same time averaged velocity $v<<c$ ). Rigid flat thin acoustic blades of width $\Lambda$ and on distance $\Lambda$ from each other parallel to the edges of the flat walls at a distance (gap) $g<<\Lambda$ and run at a speed $V<<c$. It is easy to verify that each plane waveguide is open during a time interval of duration $T$ and closed (inverter) during the time interval of the same duration $T$. The condition of synchronism $\Lambda / V=L / c=T$. Opening and closing of each waveguide is fulfilled during the time $d / V$ and very quickly relating to the time of inversion $T$, i.e. $d / V<<T$. Now it is easy to notice that for $d<<L$ and with a small thickness of the boundary layer in the flow inside the waveguide (compared to the waveguide width), the waves in each waveguide will be one-dimensional and without dispersion and with speed (this were required above). In the 
three-dimensional version of the problem, acoustic blades can be represented by the petals of two spinners rotating in phase near the waveguide bundle (see Figure 5(c)). The spinner doesn't work with the gas flow (low power for rotation), since its petals are normal to the flow and move parallel to themselves. The difference $\bar{p}-\overline{\bar{p}}>0$ in the mean (in time and in cross section) gas pressure at the inlet $\bar{p}$ and outlet $\overline{\bar{p}}$ (Figure 5(c)) pushes the gas. Finally we can formulate the following hierarchy of scales for ASBG: $\lambda_{\mathrm{w}}>\ell_{\mathrm{D}}>L \gg>\Lambda \gg d \gg \mathrm{g} \gg>\delta$, where $\lambda_{\mathrm{w}}$ wavelength of sound in the flow, $\ell_{\mathrm{D}}$-dimension of muffler, $L$-length of waveguides, $\Lambda$-wideness of blades, $d$-cross dimension of waveguide, $g$-gap between blades and waveguides, $\delta$-mean free path of gas molecules. Running blades do not produce high frequency sound (on the frequencies $\sim V / d)$. Above described parametric system equally blocks the propagation of sound from left to right, and from right to left.

\section{Experimental Testing of the Algorithm ABCM}

The goal is to reduce the ringingness of a tank (as a resonator for surface water waves) without increasing the viscosity of waveguiding media (water). In the traditional case of time constant parameters (Section 1, Figure 1(a)) of wave-suppressing devices, their dimensions are not less than basin length $\ell$. The algorithm ABCM (Section 4) was experimentally tested [3] in application to surface water waves in a tank with a length $\ell=1.5 \mathrm{~m}$ and a filling depth $h=0.22 \mathrm{~m}$ (see Figure 6(a)). The setup was conceived as an attempt to simulate the above-described one-dimensional boundary acoustic problem for a boundary with a modulated reflection coefficient, despite the two-dimensionality and dispersion of surface water waves.

\subsection{Description of the Experimental Setup}

On the left edge of the tank (Figure 6(a)) there is a wall $\mathrm{L}$ that can freely rotate around an axis located at the bottom. The wall $\mathbf{L}$ hermetically (soft corrugations) separates the water of the tank and the air on the left side.

The vertical shift $H(t)$ of the free surface of the water near the wall $\mathbf{L}$ is measured by a sensor in the form of a float. The friction breaker $\mathbf{B}(t)$ is switching periodically (with an interval $T / 2=1 / 2 \mathrm{f}_{\mathrm{M}}$, where $\mathrm{f}_{\mathrm{M}}$ the frequency of binary modulation) between two states: (a) "stopped" state $(\mathbf{B}(t)=1$, the breaker strongly pressing to the upper edge $\mathbf{L}$ and fixes the angle of deviation

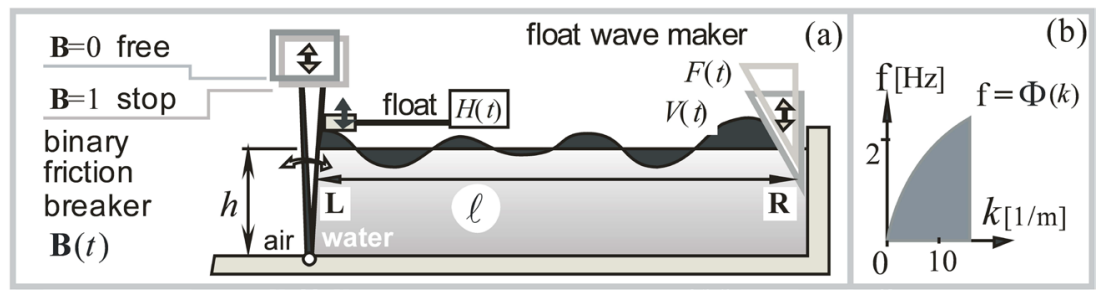

Figure 6. Draw of experiment (a) and dispersion characteristic (b) of the waves in the tank. 
of the wall $\mathbf{L})$; (b) "free" state ( $\mathbf{B}(t)=0$ the breaker does not touch the upper edge $\mathbf{L}$ ) states of the wall $\mathbf{L}$. Hydrostatic pressure on the wall $\mathbf{L}$ on the right is compensated by a soft elastic spring; the softness of spring is such that frequency $\mathrm{f}_{0}$ of free (at $\mathbf{B}(t)=0$ ) oscillations of the wall $\mathbf{L}$ is less than the frequency of the water wavelength $\lambda=2 \ell$ (seiche). On the right edge of the tank near the vertical rigid wall $\mathbf{R}$ there is a weightless (compared to the weight of the displaced water) float, to which the electromagnetic force $F(t)$ of the wave-generator is applied. The mechanical impedance of the electric drive of the wave maker is negligible compared to the impedance of the mass of water displaced by the float. The force $F(t)$ and speed $V(t)$ of the vertical displacement of the float are measured by appropriate sensors. The dispersion (frequency $\mathrm{f}[\mathrm{Hz}]$ as a function $\mathrm{f}=\Phi(k)$ of the wave number $k[1 / \mathrm{m}])$ of the propagating waves is shown in Figure 6(b).

\subsection{Pulse Drive Excitation}

There two experiments with pulsed excitation (Figure 7(a)) were made: 1) excitation of the tank (at moment $t=t_{0}$ ) by the pulse of the waveproducing force $F(t)$ with the breaker switched off $(\mathbf{B}(t)=0)$ and with the registration of damped oscillations $H(t)=H_{0}(t)$ of the free surface of the water near the wall L (Figure 7(b)); 2) excitation of the tank (at the moment $t=t_{0}$ ) by the same impulse of the wavemaker force $F(t)$, but with the breaker switched on $(\mathbf{B}(t)=(0 / 1))$ and with the recording of damped oscillations $H(t)=H_{1}(t)$ (Figure $7(\mathbf{b}))$. As can be seen from Figure $7(\mathbf{b})$, modulation $(\mathbf{B}(t)=(0 / 1))$ of the wall $\mathbf{L}$ parameters leads to a significant decrease in the wave damping. Since the waves in the tank have dispersion characteristic $\mathrm{f}=\Phi(k)$ of propagating waves, the time of one run of wave along the tank (Figure 6(a)) is estimated using the maximum group velocity $c_{\mathrm{g}}=(2 \pi)[d \Phi / d \mathrm{k}]$ of the waves as $\ell /\left[c_{\mathrm{g}}\right]_{\max }=0.65 \mathrm{~s}$ according to the graph presented in Figure $6(\mathrm{~b})$, where $\left[c_{\mathrm{g}}\right]_{\max } \approx 1.88 \mathrm{~m} / \mathrm{s}$ (i.e. at $k=0$ ).

\subsection{Sinusoidal Excitation of the Tank}

The wavemaker on the right wall $\mathbf{R}$ (Figure $7(\mathbf{c})$ ) turns on at the moment $t=0$ and produces a sinusoidal force $F(t)$ (at a frequency $\mathrm{f}_{\mathrm{W}}$ ), which is applied to the float, and the breaker $\mathbf{B}(t)$ remains off $(\mathbf{B}(t)=0$, the wall $\mathbf{L}$ is free). By the moment $t=t_{0} \gg>1 / \mathrm{f}_{\mathrm{w}}$ the stationary field of standing waves at a frequency $\mathrm{f}_{\mathrm{w}}$ is set in the tank and corresponds to a circular trajectory $[F(t), V(t)]$ in the plane (see Figure $7(\mathrm{~d})$ ). The circular impedance trajectory $[F(t), V(t)]$ corresponds to the reactive wave impedance of the wavemaker and, accordingly, weak radiation and weak absorption of waves in the tank (the phases of force $F(t)$ and velocity $V(t)$ have shift $\pi / 2$ relative to each other or the wall $\mathbf{L}$ reflection coefficient as $|\mathbf{R}(t)|=1)$. On the moment $t=t_{0}$, the breaker $\mathbf{B}(t)$ is switching on $(\mathbf{B}(t)=(0 / 1))$ at the frequency $\mathrm{f}_{\mathrm{M}}$. As a result we have frequency conversion by parametric wall into rapidly decaying 


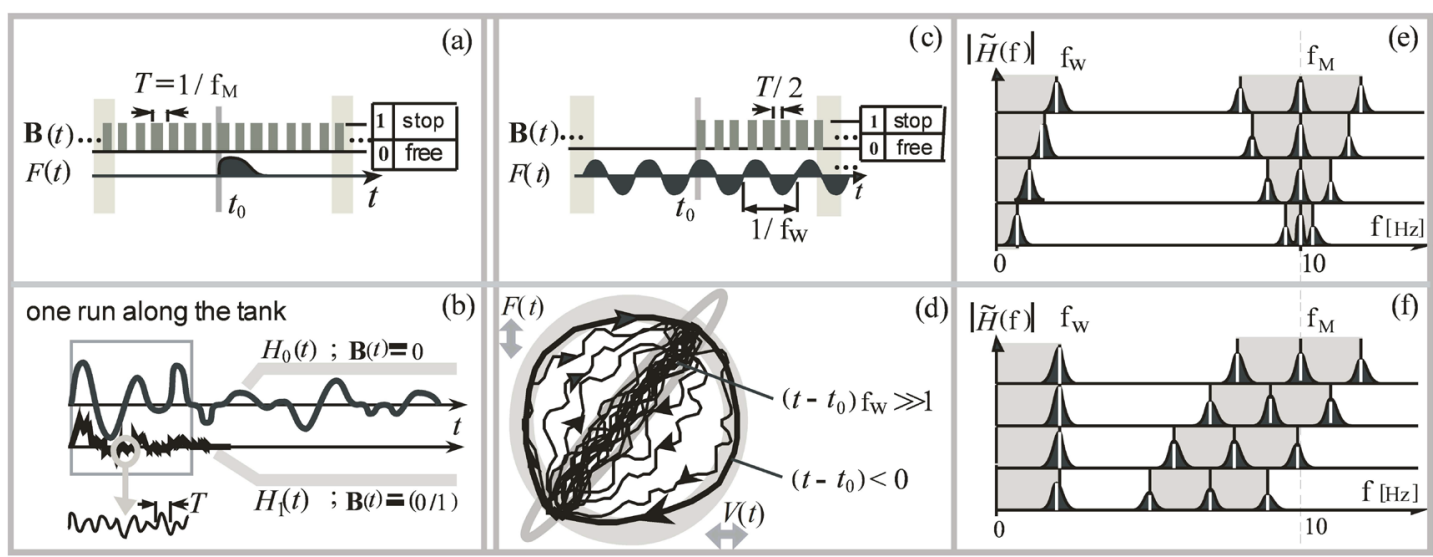

Figure 7. Pulse excitation ((a), (b)) of the tank: oscillations of the float near the left wall, when the modulation is off, oscillations of the same float when the modulation is switched on. Experiment with sinusoidal excitation of the tank (c). Evolution of the phase trajectory after breaker switching on (d). Scanning of the wavemaker frequency at a constant modulation frequency (e). Scanning of the modulation frequency at a constant wavemaker frequency (f).

high-frequency waves at combination frequencies $f_{M} \pm f_{W}, f_{M} \pm 2 f_{W}, \ldots$ Trajectory $[F(t), V(t)]$ evolutes into a straight line. This means that the wall $\mathbf{L}$ represents, from the point of view of the wavemaker, a purely active load absorbing the waves it produces. The fact of the frequency conversion of the wave field by a parametric wall $\mathbf{L}$ is clearly illustrated by measurements of the modulus $|\tilde{H}(\mathrm{f})|$ of the Fourier spectrum $\tilde{H}(\mathrm{f})$ of the oscillations $H(t)$ of the height of the float near the wall $\mathbf{L}$. Figure 7(e) shows the value $|\tilde{H}(\mathrm{f})|$ in the case of scanning the frequency $f_{w}$ of the wavemaker, and Figure $7(f)$ presents the case of scanning the modulation frequency $\mathrm{f}_{\mathrm{M}}$. At ideal modulation of the wall $\mathbf{L}$ reflection coefficient $(\mathbf{R}(t) \approx \pm 1)$, there should be no components of the spectrum $|\tilde{H}(\mathrm{f})|$ at the modulation frequency $\mathrm{f}_{\mathrm{M}}$.

\section{Conclusion}

The algorithms described in the article are constructed for the temporal representation of a boundary value problem. The presented algorithms are based on the use of high spatial-temporal resolution for fast switching wave regimes and don't require the accumulation of information about wave fields and the boundary problem, using either only instantaneous field measurements or without them. The payment for smallness of information on the fields to be damped and the boundary problem in algorithm is high-frequency radiation. Above breaking algorithms cannot be reduced to either continuous representations (partial differential equations) or traditional discrete ones (point-like wise in space or (and) in time).

\section{Conflicts of Interest}

The author declares no conflicts of interest regarding the publication of this paper. 


\section{References}

[1] Budak, B.M., Samarskii, A.A. and Tikhonov, A.N. (1964) A Collection of Problems on Mathematical Physics. Sneddon, I.N., Stark, M. and Ulam, S., Eds., Pergamon, $782 \mathrm{p}$.

[2] Widrow, J.M. and McCool, A. (1976) Comparison of Adaptive Algorithms Based on the Methods of Steepest Descent and Random Search. IEEE Transactions on Antennas and Propagation, AP-24, 615-637. https://doi.org/10.1109/TAP.1976.1141414

[3] Arabadzhi, V.V. (2011) Solutions to Problems of Controlling Long Waves with the Help of Micro-Structure Tools. Bentham Science Publishers.

https://doi.org/10.2174/97816080527521110101 